\title{
ENDOVASCULAR TREATMENT OF CHRONIC TOTAL OCCLUSION OF SUPERFICIAL FEMORAL ARTERY USING RETROGRADE APPROACH FROM POPLITEAL ARTERY
}

Nguyen Viet Lam, Phan Anh Khoa, Nguyen Ngoc Son, Ho Anh Binh

Department of Emergency and Cardiology Interventional, Hue Central Hospital

DOI: $10.47122 / v j d e .2020 .40 .5$

\begin{abstract}
An 55 years-old man with intermittent claudication was admitted to Hue central Hospital. Doppler ultrasound and digital subtraction angiography showed his left superficial femoral artery (SFA) was chronic total occlusion (CTO), and we performed endovascular treatment (EVT). We advanced a guide-wire by contralateral transfemoral approach, but guide-wire could not penetrate the occluded SFA lesion antegradely. Thus, we tried to approach the CTO lesion retrogradely. A guide-wire passed from popliteal artery to distal SFA. With a reversecontrolled antegrade and retrograde approach technique, we were able to pass the CTO lesion. Finally, two stents were implanted. We experienced a case in which retrograde approach via collateral channel from popliteal artery was effective to CTO of SFA.

Key words: chronic total occlusion (CTO), endovascular treatment (EVT), retrograde approach, superficial femoral artery (SFA), popliteal artery.

Main correspondence: Nguyen Viet Lam

Submission date: $4^{\text {th }}$ May 2020

Revised date: $15^{\text {th }}$ May 2020

Acceptance date: $26^{\text {th }}$ June 2020

Email: lamqt95@gmail.com
\end{abstract}

\section{INTRODUCTION}

The initial success rate of endovascular treatment (EVT) for chronic total occlusion (CTO) of superficial femoral artery (SFA) was reported $81^{-} 94 \%$ [1]. On the other hand, success rate for iliac artery was higher than that of SFA. In EVT for CTO of SFA, we can choose antegrade or retrograde to approach the lesion. In some cases, antegrade access can be difficult, so we must approach via retrograde pathway. We report a case of successful EVT for CTO of SFA using retrograde approach from popliteal artery.

\section{CASE REPORT}

An 55 years old man with a history of diabetes and hypertension was admitted to Hue central hospital complaining intermittent claudication of bilateral lower limbs within 100 meter, a Rutherford classification of grade I-3. He was given Cilostazole, Aspirin, Rosuvastatin, Insulin and Perindopril. His right ankle brachial index (ABI) was 0.8 and left ABI was 0.55. Doppler ultrasound and digital subtraction angiography demonstrated that his left SFA were CTO and collateral channels developed from left deep femoral artery to SFA distal (Fig.1B). His symptom was not improved by medications. We discussed with cardiovascular surgeons regarding indication of resvascularization therapy. Comparing femoro-popliteal ( $\left.\mathrm{F}^{-} \mathrm{P}\right)$ bypass using the vascular graft, it has been reported that the initial success rate and secondary patency rate of EVT are close to F$\mathrm{P}$ bypass. Furthermore, the overall complication is higher in the F-P bypass than in EVT in claudication patients with Trans Atlantic Inter - Society Consensus (TASC) II $\mathrm{C}$ and $\mathrm{D}[1]$. 


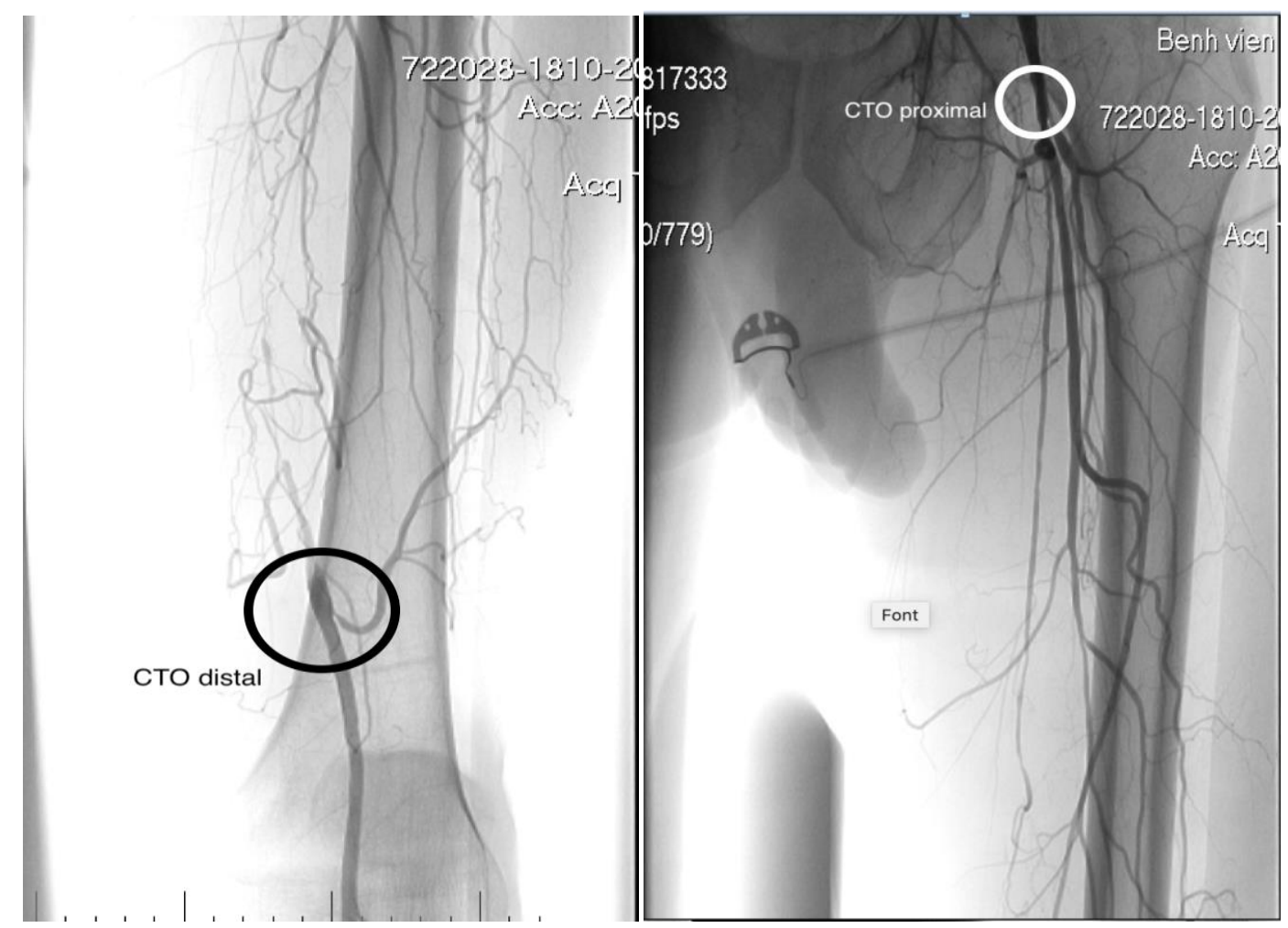

Fig.1A

Fig.1B

Fig.1. Angiography of left superficial femoral artery A: Angiography showed chronic total occlusion in the proximal potion of left SFA B: Collateral channels devoleped from deep femoral artery to distal CTO of left SFA

We performed EVT to CTO of left SFA. A $6 \mathrm{~F}$ Sheath was inserted in the right femoral artery. Angiography showed total occlusion in the proximal potion of left SFA and collateral channels developed from DFA to distal potion of CTO of SFA. We used 0.010" guide wire and 0.009 " guide wire, but the guide -wires did not reach distal true lumen. Since the antegrade injection demonstrated some collateral channels from DFA to the distal CTO and reperfusion flow at one-third below of SFA. So, we approach to distal SFA via retrograde technique from left popliteal artery. We putted 6F sheath into the left popliteal artery, then, we used 0.035 " straight guide wire with Multipurpose $4 \mathrm{~F}$ catheter passed into from distal to proximal SFA. We performed antegradeballon dilation with ballon angioplasty $5.0 \times 120 \mathrm{~mm}$ in the proximal SFA to distal SFA. Then, angiography showed revascularization flow in the proximal ton distal of SFA and stenosis of SFA about $40-50 \%$. We were implanted with stent self-expanding $7.0 \times 170 \mathrm{~mm}$ in the proximal of SFA and stent $6.0 \times 150 \mathrm{~mm}$ in the distal of SFA.

We performed post balloon dialation with balloon 6.0 x $120 \mathrm{~mm}$ with pressure $10 \mathrm{~atm}$. The final angiography showed well expanded stents. All target lesions were successfully dilated in 2 hours. His left leg reduce pain and claudication. 


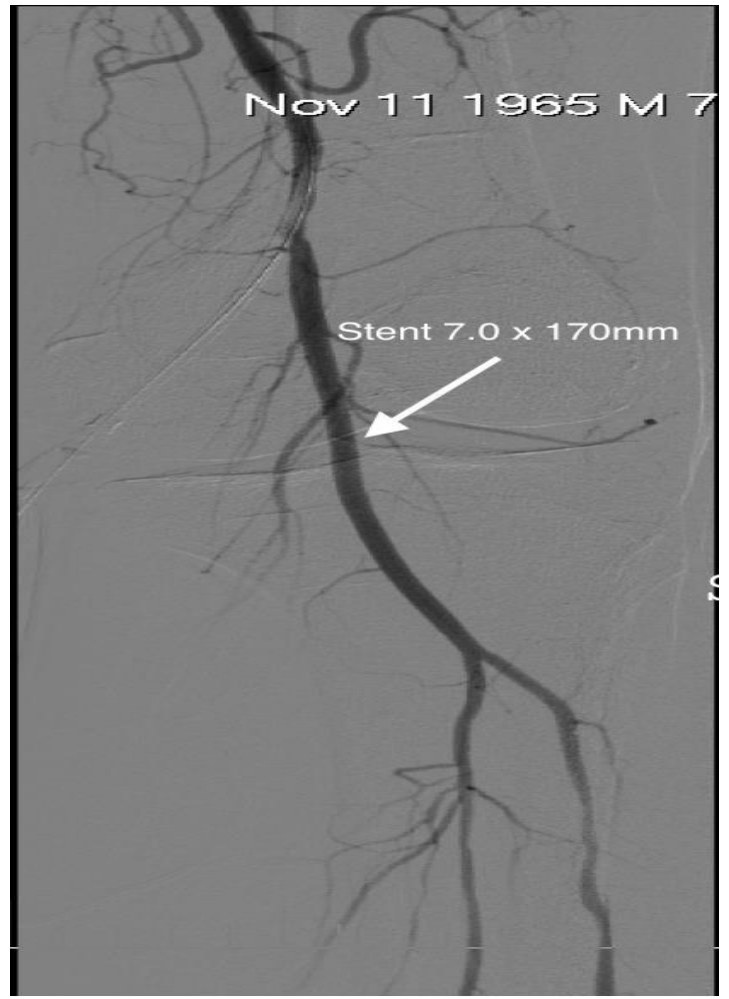

Fig.2.A

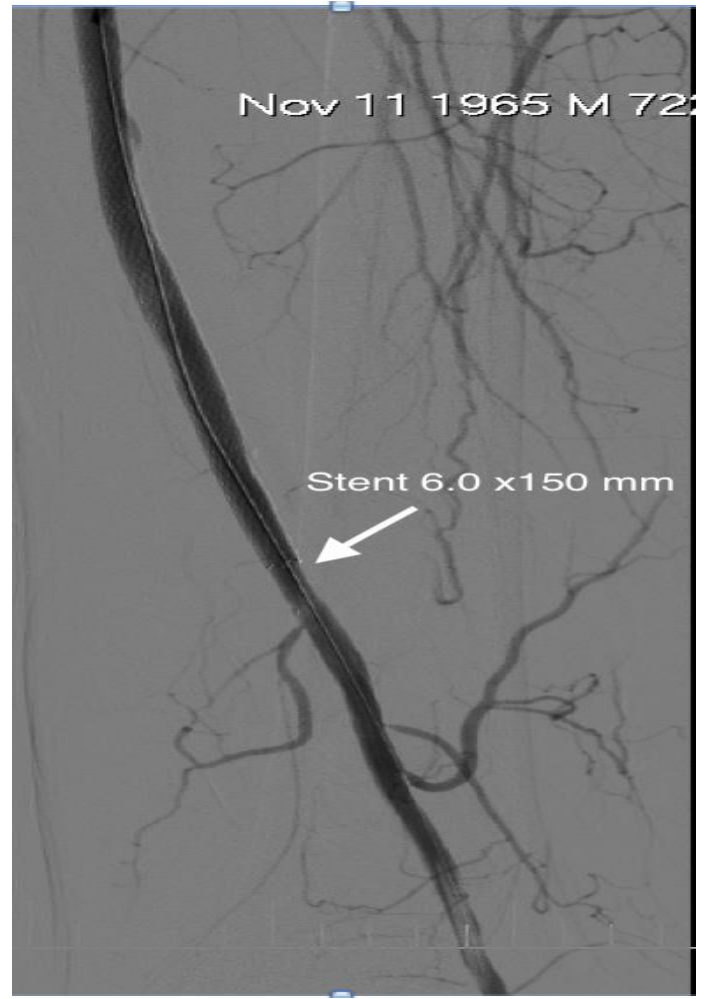

Fig.2.B

Fig.2. Implanted self-expanding stents

A: Implanted stent $7.0 \times 170 \mathrm{~mm}$ in the proximal of left SFA

B: Implanted stent $6.0 \times 150 \mathrm{~mm}$ in the distal of left SFA. Final angiography showed well expanded stents.

\section{DISCUSSION}

The patients with TASC D lesion of SFA such as this patient are recommended to be treated by surgical femoral popliteal bypass [2]. However, since it has been recently reported that device and technical developments provide high recanalization rates [3]. Comparing with $\mathrm{F}^{-} \mathrm{P}$ bypass, in a recent report, the 1 year and 5 years primary patency rates were $82.1 \%$ and $69.4 \%$ in the bypass surgery and $67.8 \%$ and $45.2 \%$ in the EVT in claudication patients with TASC C and $\mathrm{D}$, respectively. However, the 1 year and 5 years secondary patency rates were $93.2 \%$ and $79.5 \%$ in the $\mathrm{F}-\mathrm{P}$ bypass surgery and $90.1 \%$ and $85.1 \%$ in the EVT, respectively. Although the bypass surgery group had a higher primary patency rate than the EVT, secondary patency rates did not differ significantly between the EVT and bypass surgery. Such patients treated with EVT have been increasing. Chronic total occlusion of the superficial femoral artery is a femoropopliteal disease characterized by long, diffuse occlusions and/or long total occlusions, and it commonly requires endovascular treatment.

The primary success rate of EVT for SFA CTO has improved from $75 \%$ in 2001 to $81 \%-94 \%$ in 2014. EVTs for SFA are generally managed by the antegrade approach, which uses a contralateral retrograde puncture or ipsilateral antegrade puncture of the common femoral artery [4]. Alternatively, the popliteal approach is less frequently utilized. In this case, the popliteal approach performed after the antegrade approach was unsuccessful and the lesion had 
severe calcification and/or CTO length was relatively long.

\section{CONCLUSIONS}

Retrograde revascularization via popliteal artery approach is effective for CTO of superficial femoral artery when failed with antegrade approach.

\section{REFERENCES}

1. Aihara H, Soga Y, Mii S, et al.LongTerm Outcome After Endovascular Therapy Versus Bypass Surgery in Claudication Patients With Trans ${ }^{-}$ Atlantic Inter- Society Consensus- II C and D Femoropopliteal Disease. Circ J, 78 : 457- 464, 2014.

2. Norgren L, Hiatt WR, Dormandy JA, et al. Inter- Society Consensus fot the Management of Peripheral Arterial Disease (TASC II). Eur J Vasc EndovascSurg, 33 : S1-75, 2007

3. Suzuki K, lida O, Soga Y, et al. Longterm results of the S.M.A.R.T Control (TM) stent for superficial femoral artery lesions, J-SMART registry. Circ J, 75: 939-944, 2011.

4. Wei, L. M., Zhu, Y. Q., Zhang, P. L., Lu, H. T., \& Zhao, J. G. (2018). Integrated application of antegrade and retrograde recanalization for femoral-popliteal artery chronic total occlusions: outcomes compared with antegrade recanalization. Quantitative imaging in medicine and surgery, 8(6), 568-578. https://doi.org/10.21037/qims.2018.07.02 